\title{
Comments on: Static and dynamic source locations in undirected networks
}

\author{
Martine Labbé ${ }^{1}$
}

Published online: 25 September 2015

(C) Sociedad de Estadística e Investigación Operativa 2015

Network location problems consist in selecting a subset of facility locations (usually a subset of vertices of the network) to serve a given set of demand points. The location decisions and the allocation of demand points to open facilities are usually based on operating costs and on the distances separating the demand points and the open facilities, those distances being given by shortest path lengths, see e.g. Laporte et al. (2015).

In the problems addressed in the paper "Static and Dynamic Source Locations in Undirected Networks", a different very interesting paradigm is considered by requesting that sufficient flow can be send from open facilities to each vertex or demand point. In other words, the studied location problems involve maximum flow values instead of shortest path distances. Given a capacitated network, the motto here is to locate facilities (sources) from which the maximum flow to each vertex is at least as larger as its demand. A first part of the paper is devoted to the single cover problem, in which the demand of each vertex must be satisfied independently. It is shown that the problem can be solved with a greedy algorithm. To this end, the authors highlight nicely that the problem presents a matroid structure. When the demand of all vertices of the network must be satisfied simultaneously, the problem becomes NP-hard, but the authors identify variants which remain polynomial by restricting to tree networks.

This comment refers to the invited paper available at doi:10.1007/s11750-015-0395-7.

The author's research is funded by the Interuniversity Attraction Poles Programme initiated by the Belgian Science Policy Office.

$\bowtie$ Martine Labbé

mlabbe@ulb.ac.be

1 Computer Science Department, Université Libre de Bruxelles, Brussels, Belgium 
Finally, the authors address the single cover problem in dynamic networks, i.e., in which each vertex must receive, over a given horizon, a maximal dynamic flow whose value is at least as large as its demand. The problem is shown to be NP-hard and some polynomial cases are discussed.

The paper is nicely structured and the presentation of the results is particularly didactic. Further, the results are appropriately situated within the existing literature on the subject. Maybe, more details about applications of the problems considered would have made the paper even more attractive to the readers of TOP.

The idea of replacing the concept of shortest path by the one of minimum flow is very interesting and open various avenues of research. My aim here is to address some of them.

First, one could consider problems in which a simple optimization problem other than the shortest path or maximum flow could be considered to define the feasibility of set of facility locations.

Consider the following Covering Problem (CP). Given a matrix $D=(d(v, w))$ of distances between every pair of vertices $v, w \in V$ and a fixed cost $f_{v}$ and a radius $R_{v}$ for each $v \in V$, open a subset $S$ of vertices (facilities) with minimum total fixed cost and such that, for each vertex $v$, there exists an open facility $s \in S$ such that $d(s, v)$ is smaller than or equal to $R_{v}$.

$$
\begin{array}{ll}
\min & \sum_{s \in S} f_{s} \\
\text { s.t. } & \min _{s \in S} d(v, s) \leq R_{v}, \quad \forall v \in V, \\
& S \subseteq V .
\end{array}
$$

When the distances are given by shortest path lengths, the p-Center problem can be seen as a special case of CP and it is NP-hard (Kariv and Hakimi 1979).

Now, let $\operatorname{Val}(v, w)$ represents the maximum flow between $v$ and $w, d_{v}$ the demand (in flow) of vertex $v$ and $R$ a large constant. If we set

$$
d(v, w)=R-\operatorname{Val}(v, w), \quad \forall v, w \in V
$$

and

$$
R_{v}=R-d_{v}, \quad \forall v \in V,
$$

then CP is precisely the (static) Single Cover Problem. The fact that it is now polynomial seems to follow from that fact that the maximum flow value satisfies the two properties presented in Lemma 1 of Sect. 2. Interestingly, these two conditions for $d(v, w)$ defined by (1) mean that $d(v, w)$ is an ultrametric. This last notion arises naturally in the context of phylogenetics, see e.g. Huson et al. (2010) and Semple and Steel (2003).

This observation raises the following two questions: is the problem CP polynomially solvable for any ultrametric, and, what are the other combinatorial optimization problems that would lead to an ultrametric?

The second avenue of research suggested by the paper under consideration consists in variants of location problems with maximum flow requests. One may indeed be 
interested in a p-Median version of the problem in which the goal is to maximize the total flow that can be send to the vertices, or to maximize the number of vertices whose demand can be fully satisfied. One may also consider similar variants in which fixed costs have to be paid for opening facilities rather than minimizing their number. Which of these problems are NP-hard? Are there some polynomial special cases? How to formulate and solve the difficult ones?

My feeling is that this field of research is wide open and promising.

\section{References}

Huson DH, Rupp R, Scornavacca C (2010) Phylogenetic networks: concepts, algorithms and applications. Cambridge University Press, Cambridge

Kariv O, Hakimi SL (1979) An algorithmic approach to network location problems. I: The p-centers. SIAM J Appl Math 37(3):513-538

Laporte G, Nickel S, Saldanha da Gama F (2015) Location Science. Springer, Berlin

Semple C, Steel MA (2003) Phylogenetics, vol 24. Oxford University Press, Oxford 